Weingartenschen Gleichungen in der Theorie der Strahlensysteme. 14. Spezielle Sätze und Aufgaben der Flächentheorie.

Nun etwas über den Charakter des Werkes. Der Titel „Grundlagen“ der Differentialgeometrie verleitet $\mathrm{zu}$ der Annahme, der Verfasser werde bestrebt sein, mit mathematischer Klarheit und Strenge die manchmal etwas verschwommenen und nebelhaften Begriffsbildungen dieses Zweiges der Geometrie festzulegen, der bisher aus seinem heroischen Stadium noch kaum herausgetreten ist. In dieser Meinung bestärkt auch ein Passus im Vorwort: " . obgleich die Differentialgeometrie noch in ihren Anfängen steckt". Aber schon in der ersten Seite des Textes wird man überzeugend eines Besseren belehrt durch die Festsetzung, die da getroffen wird: , . . . mit Rücksicht darauf sei ein für allemal bemerkt, daß von dem Funktionensystem immer alle die Eigenschaften vorausgesetzt werden sollen, deren Einführung der Gang der jeweiligen Untersuchung als notwendig oder zweckmäBig erscheinen läßt.“

Das Hauptgewicht ist auf die formale Seite der Rechnungen gelegt, die die Methoden von Ricci benutzen. Als Hilfsmittel der Rechnung werden insbesondere "geometrische Differentiationen“ verwendet, das heißt nichts anderes als was der Physiker als Differentiation einer Funktion in einer gegebenen Richtang bezeichnet.

Wesentlich neue Ergebnisse geometrischer Natur scheinen zu fehlen. Figuren sind nur recht spärlich vorhanden.

Wilhelm Blaschke.

\title{
Geschichte des naturwissenschaftlichen und mathematischen
} Unterrichts. 'Von Franz Pahl, Leipzig, Quelle u. Meyer, 1913.

Das vorliegende Werk (368 Seiten) bietet weit mehr als der Titel verspricht - nicht etwa nur eine Geschichte der Lehrverfassung und Lehrmethoden, sondern, soweit es die vorwiegend pädagogischen Absichten des Verf. verlangen, eine Geschichte der Mathematik und der Naturwissenschaften, vorwiegend der exakten, überhaupt. Trotz der durch den großen Umfang des Gebietes erforderten Kürze ist die Darstellnng keineswegs schematisch, sondern durchaus flüssig und anregend, umsomehr als der Stand der naturwissenschaftlichen Forschung und Lehre stets im Zusammenhang mit dem ganzen Kulturbild gegeben wird, wie jedes Zeitalter es uns hinterlassen hat. Die Entwicklung der mathematisch-naturwissenschaftlichen Kenntnisse wird von den frühesten Zeiten an beschrieben, die des Unterrichts in fünf Kapiteln: Klosterschulen and Universitäten im Mittelalter, die Gelehrtenschulen des humanistischen Zeitalters, die pädagogische Epoche von Comenius und Leibniz, der mathematisch-naturwissenschaftliche Unterricht des 18. Jahrhunderts, endlich die Reformbewegung im 19, die mit Herbart beginnt and seit kurzem mit frischer Stärke eingesetzt hat. - Das Buch von Pahl ist für Lehrerbibliotheken zu empfehlen und wird besonders dem Lehrer der. Mathematik wertvoll sein.

$F$.

Betrachtungen über mathematische Erziehung. Von B. Branford. Übersetzt von R. Schimmack und H. Weinreich. Teubner, 1913.

Der in diesem Buch mit großer Geduld und Breite auf alle Stufen des mathematischen Unterrichts angewendete Grundsatz ist kurz der: Die Ent- 